\title{
Food, Drugs, and Medical Devices Regulatory Document
}

National Cancer Institute

\section{Source}

National Cancer Institute. Food, Drugs, and Medical Devices Regulatory Document. NCI

Thesaurus. Code C73110.

A regulatory document encompassed by or derived from the CFR T itle 21. 